\title{
International Journal of Antimicrobial Agents
}

\section{Emergence of Haemophilus parainfluenzae resistant to third-generation}

cephalosporins in Italy: potential role of PBP3 and PBP5 substitutions in high-level resistance

--Manuscript Draft--

\begin{tabular}{|l|l|}
\hline Manuscript Number: & IJAA-D-20-00744R3 \\
\hline Article Type: & Letter (to the Editor) \\
\hline Corresponding Author: & $\begin{array}{l}\text { Luigi Principe, M.S. } \\
\text { Presidio Ospedaliero Alessandro Manzoni } \\
\text { ITALY }\end{array}$ \\
\hline First Author: & Luigi Principe, M.S. \\
\hline Order of Authors: & Luigi Principe, M.S. \\
\hline & Odette J Bernasconi \\
\hline Valentina Viaggi \\
\hline Edgar I Campos-Madueno \\
\hline Andrea Endimiani \\
\hline
\end{tabular}


To Jean-Marc Rolain,

September 2, 2020

\section{Editor in Chief}

International Journal of Antimicrobial Agents

Enclosed please find the revision (R3) of the manuscript entitled "Emergence of Haemophilus parainfluenzae resistant to third-generation cephalosporins in Italy: potential role of PBP3 and PBP5 substitutions in high-level resistance”, which I am re-submitting as a Letter to International Journal of Antimicrobial Agents.

The text has been revised, as required by the Section Editor. I am providing the files with the highlighted text, a clean copy and the point-by-pint reply to Reviewers' comments.

The revised manuscript has been seen and approved by all the authors. The material is original, unpublished, and has not been simultaneously submitted to another medical journal.

We hope that the information provided with this manuscript will be interesting to the audience of International Journal of Antimicrobial Agents, and that it will meet with the Editor's approval. We look forward to hearing from you at your earlier convenience.

Yours sincerely,

Luigi Principe

Please, address the correspondence concerning this manuscript to:

Luigi Principe, M.S.

Mailing address: Microbiology and Virology Unit

A. Manzoni Hospital

Via dell'Eremo, 9/11 - 23900 - Lecco, Italy

Phone: +390341 489630

Fax: +390341 489601

E-mail: luigi.principe@gmail.com 


\section{POINT BY POINT REPLY TO REVIEWERS' COMMENTS}

\section{Section Editor:}

SPAdes, Canu, and ParSNP have been published in peer-reviewed journal and their authors should get credit for using the product of their research. As previously requested, please insert regular citations to published articles for all these bioinformatics tools, even if the number of citations then exceeds the usual limit for letters.

Reply: References have been added (\#6, \#7, \#8), as required. 


\section{HIGHLIGHTS}

- The spread of resistance traits in $H$. parainfluenzae is a matter of concern

- Here we describe the first case of resistance to 3-GCs in Haemophilus spp. in Italy

- Several AA substitutions in PBP3 and PBP5 were detected in 3-GCs-resistant isolate

- AA substitutions in PBP5 were not so far reported in 3-GCs-resistant Haemophilus spp.

- Empirical therapy without microbiological investigations could be harmful to patients 

0 1 2

Emergence of Haemophilus parainfluenzae resistant to third-generation cephalosporins in Italy: potential role of PBP3 and PBP5 substitutions in high-level resistance Luigi Principe $^{1 *}$, Odette J. Bernasconi ${ }^{2}$, Valentina Viaggi ${ }^{1}$, Edgar I. Campos-Madueno², Andrea Endimiani ${ }^{2}$, Francesco Luzzaro ${ }^{1}$

${ }^{1}$ Clinical Microbiology and Virology Unit, A. Manzoni Hospital, Lecco (Italy)

${ }^{2}$ Institute for Infectious Diseases, University of Bern (Switzerland)

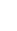
3 4

\section{*Corresponding author:}

6 Luigi Principe, M.S.

17 Mailing address: Microbiology and Virology Unit, A. Manzoni Hospital

8 Via dell'Eremo, 9/11 - 23900 - Lecco, Italy

9 Phone: +390341489630

Fax: +390341489601

1 E-mail: luigi.principe@gmail.com 
Sir,

The potential role of Haemophilus parainfluenzae as a sexually transmitted genitourinary pathogen is well recognized, even though indistinctive clinical symptoms make difficult the differentiation of urethritis due to Haemophilus spp. from other pathogens [1]. Of note, H. parainfluenzae represents an example of how a community-acquired pathogen could become a serious concern for antimicrobial treatment due to emergence of resistance traits. In particular, resistance to $\beta$-lactams emerged from the diffusion of plasmid-mediated $\beta$-lactamases (TEM-1, ROB-1). Later, isolates resistant to third-generation cephalosporins (3-GCs) have been sporadically reported worldwide, mostly showing alterations in the transpeptidase domain of penicillin-binding protein 3 (PBP3), with variable phenotypic resistance profiles depending on the specific amino acid (AA) substitution patterns [2]. Resistance to carbapenems is considered exceptional [2-4], and so far, not reported in H. parainfluenzae.

With regard to Italy, though surveillance data are scarce and not updated, resistance to 3-GCs has never been reported. Here, we describe the first Italian 3-GC-resistant $H$. parainfluenzae isolated from a genitourinary infection and the related molecular investigation using whole genome sequencing (WGS).

In 2018, a 45-years-old Italian male with purulent urethral discharge was examined for sexually transmitted diseases. Anamnestic consultation revealed that he had unprotected sex with a woman of Serbian origin. A 7-day treatment with ceftibuten (400 mg, once daily) was initially prescribed as empirical therapy. At the end of this treatment, due to the persistence of clinical symptoms (urethral pain, dysuria, purulent urethral discharge), a microbiological culture from urethral swab was performed at the Microbiology Laboratory of A. Manzoni Hospital (Lecco, Italy).

Bacterial identification and antimicrobial susceptibility testing were performed using MALDI-TOF MS (VITEK MS, bioMérieux, Marcy l'Etoile, France) and broth microdilution method (YITHMN panel, Thermo Fisher Scientific, Waltham, MA, USA), respectively. Furthermore, Etest (bioMérieux) and CLSI standard broth microdilution methods were also performed for antibiotics 
and/or MICs not included in the YITHMN panel. Production of $\beta$-lactamases was investigated by nitrocefin-based test (Thermo Fisher Scientific). MICs were interpreted according to the EUCAST (v. 9.0) breakpoints recommended for H. influenzae (www.eucast.org).

As a result, an $H$. parainfluenzae (LC/1315.18) negative for $\beta$-lactamase production but resistant to penicillin, ampicillin, amoxicillin/clavulanate, cefotaxime, ceftriaxone was identified. The isolate also showed a non-susceptible profile for doxycycline and a borderline MIC for tetracycline. Fortunately, LC/1315.18 remained susceptible to carbapenems (though meropenem showed a borderline MIC of $2 \mathrm{mg} / \mathrm{L}$ ), trimethoprim/sulfamethoxazole and ciprofloxacin (Table 1). Thus, ciprofloxacin (500 mg, bid) was administered for 10 days. The new antimicrobial treatment was successful in eradicating the infection, as demonstrated by culture follow-up analysis.

Since the antibiotic resistance profile of LC/1315.18 was exceptional, WGS was conducted with both NovaSeq (Illumina Inc.) and MinION (Oxford Nanopore) platforms as previously done [5]. Assemblies of the Illumina whole-genome shotgun and Nanopore hybrid assemblies were done with SPAdes (v.3.12.0) and Canu (v1.7), respectively [6-7]. All assemblies were deposited to GenBank under BioProjects PRJNA515889 (Nanopore hybrid) and PRJNA575148 (Illumina SPAdes). AA sequences of PBP3 and PBP5 were compared to their wild type counterparts of $H$. parainfluenzae T3T1 strain (GenBank accession no. NC_015964) by implementing the protein database Protein BLAST (blastp) of NCBI. The phylogenetic tree was constructed using the tool for microbial core genome alignment and variant detection Parsnp (v1.2; http://github.com/marbl/harvest) [8]. In short, a core-genome alignment was generated with the genomes of $26 \mathrm{H}$. parainfluenzae (WGS available in the GenBank nucleotide database) and the Illumina SPAdes assembly of LC/1315.18. Finally, the FigTree software (v1.4.4; http://tree.bio.ed.ac.uk/software/figtree) was implemented to visualize the Parsnp core-genome SNV phylogenetic tree.

WGS analysis showed several point mutations in PBP3 and PBP5 sequences, thus suggesting the potential role of derived AA substitutions in determining high-level resistance to 3-GCs. Of note, PBP3 showed 98\% AA identity (Supplemental Fig. 1) with respect to the wild type $H$. 
parainfluenzae strain T3T1 (GenBank accession no. FQ312002) (i.e., Gly11Ala; Val34Met; Val356Leu; Ser385Thr; Ile442Phe; Val511Ala; Ile519Val; Asn526Lys; Asp551Leu; Thr574Ala; Phe602Leu). Instead, PBP5 displayed only 91\% AA identity (Supplemental Fig. 2) due to 37 AA substitutions scattered through the entire protein sequence. Since no $\beta$-lactamases were detected, resistance to $\beta$-lactams was reasonably attributable to the substitutions in PBP3 and/or PBP5.

Comparison with other genomes deposited in GenBank showed that LC/1315.18 had a unique profile, with no clonal relationship with other previously reported $H$. parainfluenzae strains (Supplemental Fig. 3).

We described the first 3-GC-resistant $H$. parainfluenzae strain from Italy. More importantly, the isolate showed very high MIC values for 3-GCs (the one of cefotaxime was notable: >32 mg/L) and a borderline MIC for meropenem (data not previously described in $H$. parainfluenzae). Our molecular analysis showed several AA substitutions in PBP3 and PBP5 (most of them not so far described) that are probably responsible for this phenotype. However, we could only evaluate the cumulative effect of these substitutions providing this particular resistance phenotype. As a limitation, we did not explore in vitro the effect of individual polymorphisms on the resistance to $3 \mathrm{GC}$ by cloning or site mutagenesis experiments, so not assessing which substitution could have a major role for this resistant phenotype.

The spread of resistance traits in $H$. parainfluenzae is a matter of concern. To this regard, our findings highlight that empirical prescription of antibiotics in the community setting without using microbiological data could be harmful to patients. Moreover, continuous education and awareness of general practitioners toward resistance to antibiotics, including rare phenotypes, could be of utmost importance in promoting antibiotic and diagnostic stewardship, as well in the community setting. 
101 Competing interests: None declared.

102 Funding: None.

103 Ethical approval: Not required.

104 


\section{REFERENCES}

1061 Deza G, Martin-Ezquerra G, Gómez J, Villar-García J, Supervia A, Pujol RM. Isolation of 107 Haemophilus influenzae and Haemophilus parainfluenzae in urethral exudates from men with 108 acute urethritis: a descriptive study of 52 cases. Sex Transm Infect 2016; 92: 29-31.

1092 Skaare D, Anthonisen IL, Kahlmeter G, Matuschek E, Natås OB, Steinbakk M, et al. 110 Emergence of clonally related multidrug resistant Haemophilus influenzae with penicillin111 binding protein 3-mediated resistance to extended-spectrum cephalosporins, Norway, 2006 to 112 2013. Euro Surveill 2014; 19, pii: 20986.

1133 Cerquetti M, Giufrè M, Cardines R, Mastrantonio P. First characterization of heterogeneous 114 resistance to imipenem in invasive nontypeable Haemophilus influenzae isolates. Antimicrob 115 Agents Chemother 2007; 51: 3155-61.

1164 Cherkaoui A, Diene SM, Renzoni A, Emonet S, Renzi G, François P, et al. Imipenem 117 heteroresistance in nontypeable Haemophilus influenzae is linked to a combination of altered 118 PBP3, slow drug influx and direct efflux regulation. Clin Microbiol Infect 2017; 23: 118.e9-19.

1195 Bernasconi OJ, Principe L, Viaggi V, Luzzaro F, Endimiani A. Novel vanA-carrying plasmid in 120 a clinical isolate of Enterococcus avium. Int J Antimicrob Agents 2019; 53: 876-7.

1216 Bankevich A, Nurk S, Antipov D, Gurevich AA, Dvorkin M, Kulikov AS, et al. SPAdes: a new 122 genome assembly algorithm and its applications to single-cell sequencing. J Comput Biol 2012; $123 \quad 19: 455-77$.

1247 Koren S, Walenz BP, Berlin K, Miller JR, Bergman NH, Phillippy AM. Canu: scalable and 125 accurate long-read assembly via adaptive $k$-mer weighting and repeat separation. Genome Res $126 \quad 2017 ; 27: 722-36$.

1278 Treangen TJ, Ondov BD, Koren S, Phillippy AM. The Harvest suite for rapid core-genome 128 alignment and visualization of thousands of intraspecific microbial genomes. Genome Biol 129 $2014 ; 15: 524$. 


\section{LEGEND TO THE FIGURES}

132

133 Supplemental Figure 1. NCBI protein BLAST results of PBP3 (i.e., query H. parainfluenzae 134 LC/1315.18, subject H. parainfluenzae T3T1).

135

136 Supplemental Figure 2. NCBI protein BLAST results of PBP5 (i.e., query H. parainfluenzae 137 LC/1315.18, subject $H$. parainfluenzae T3T1).

138

139 Supplemental Figure 3. Phylogenetic tree based on single nucleotide variants (SNV) of $26 H$. 140 parainfluenzae WGS available in the NCBI database and LC/1315.18 strain.

141 Tree construction was performed using Parsnp (http://github.com/marbl/harvest) with HiSeq 142 paired-end reads assembled with the genome assembly algorithm SPAdes. The parameter $-c$ was 143 used for the curated genome directory, and $-C$ for the maximal cluster D value (reference coverage) 144 was set to 5000. Remaining options were let as default. Final graphical visualization of the 145 phylogenetic relationships was created with FigTree software 146 (http://tree.bio.ed.ac.uk/software/figtree). Tip labels correspond to H. parainfluenzae strains. NA, 147 not available 
Table 1: Antibiotic susceptibility profile of Haemophilus parainfluenzae (LC/1315.18)

\begin{tabular}{lcc}
\hline \multirow{2}{*}{ Antibiotic } & \multicolumn{2}{c}{ MIC values, mg/L (S, I, R) } \\
\cline { 2 - 3 } & Etest & Broth microdiluition \\
\hline Ampicillin & $24(\mathrm{R})$ & $4(\mathrm{R})$ \\
Amoxicillin/clavulanate & $2(\mathrm{R})$ & $>2 / 2(\mathrm{R})$ \\
Ceftriaxone & $>32(\mathrm{R})$ & $2(\mathrm{R})$ \\
Cefotaxime & & $>2(\mathrm{R})$ \\
Ciprofloxacin & & $0.016(\mathrm{~S})$ \\
Doxycycline & & $2(\mathrm{I})$ \\
Levofloxacin & $2(\mathrm{~S})$ & $0.06(\mathrm{~S})$ \\
Meropenem & & $2(\mathrm{~S})$ \\
Penicillin & & $>1(\mathrm{IE})$ \\
Tetracycline & & $1(\mathrm{~S})$ \\
Trimethoprim/sulfamethoxazole & & $0.12 / 2.37(\mathrm{~S})$ \\
Ertapenem & & \\
Imipenem & $0.75(\mathrm{~S})$ &
\end{tabular}

Abbreviations: MIC, minimum inhibitory concentration; R, resistant; I, intermediate; S, susceptible; IE, insufficient evidence. Since no specific criteria are currently available by EUCAST, results were interpreted according to those recommended for $H$. influenzae. 
Click here to access/download Supplementary data Supplemental Figure 1.pdf 


\section{Click here to access/download Supplementary data Supplemental Figure 2.pdf}




\section{Click here to access/download Supplementary data Supplemental Figure 3.pptx}




\section{Emergence of Haemophilus parainfluenzae resistant to third-generation cephalosporins in} Italy: potential role of PBP3 and PBP5 substitutions in high-level resistance Luigi Principe $^{1 *}$, Odette J. Bernasconi ${ }^{2}$, Valentina Viaggi ${ }^{1}$, Edgar I. Campos-Madueno ${ }^{2}$, Andrea Endimiani ${ }^{2}$, Francesco Luzzaro ${ }^{1}$

${ }^{1}$ Clinical Microbiology and Virology Unit, A. Manzoni Hospital, Lecco (Italy)

${ }^{2}$ Institute for Infectious Diseases, University of Bern (Switzerland)

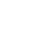

\section{*Corresponding author:}

6 Luigi Principe, M.S.

7 Mailing address: Microbiology and Virology Unit, A. Manzoni Hospital

8 Via dell'Eremo, 9/11 - 23900 - Lecco, Italy

9 Phone: +390341 489630

20 Fax: +390341489601

21 E-mail: luigi.principe@gmail.com 
Sir,

The potential role of Haemophilus parainfluenzae as a sexually transmitted genitourinary pathogen is well recognized, even though indistinctive clinical symptoms make difficult the differentiation of urethritis due to Haemophilus spp. from other pathogens [1]. Of note, H. parainfluenzae represents an example of how a community-acquired pathogen could become a serious concern for antimicrobial treatment due to emergence of resistance traits. In particular, resistance to $\beta$-lactams emerged from the diffusion of plasmid-mediated $\beta$-lactamases (TEM-1, ROB-1). Later, isolates resistant to third-generation cephalosporins (3-GCs) have been sporadically reported worldwide, mostly showing alterations in the transpeptidase domain of penicillin-binding protein 3 (PBP3), with variable phenotypic resistance profiles depending on the specific amino acid (AA) substitution patterns [2]. Resistance to carbapenems is considered exceptional [2-4], and so far, not reported in H. parainfluenzae.

With regard to Italy, though surveillance data are scarce and not updated, resistance to 3-GCs has never been reported. Here, we describe the first Italian 3-GC-resistant $H$. parainfluenzae isolated from a genitourinary infection and the related molecular investigation using whole genome sequencing (WGS).

In 2018, a 45-years-old Italian male with purulent urethral discharge was examined for sexually transmitted diseases. Anamnestic consultation revealed that he had unprotected sex with a woman of Serbian origin. A 7-day treatment with ceftibuten (400 mg, once daily) was initially prescribed as empirical therapy. At the end of this treatment, due to the persistence of clinical symptoms (urethral pain, dysuria, purulent urethral discharge), a microbiological culture from urethral swab was performed at the Microbiology Laboratory of A. Manzoni Hospital (Lecco, Italy).

Bacterial identification and antimicrobial susceptibility testing were performed using MALDI-TOF MS (VITEK MS, bioMérieux, Marcy l'Etoile, France) and broth microdilution method (YITHMN panel, Thermo Fisher Scientific, Waltham, MA, USA), respectively. Furthermore, Etest (bioMérieux) and CLSI standard broth microdilution methods were also performed for antibiotics 
and/or MICs not included in the YITHMN panel. Production of $\beta$-lactamases was investigated by nitrocefin-based test (Thermo Fisher Scientific). MICs were interpreted according to the EUCAST (v. 9.0) breakpoints recommended for H. influenzae (www.eucast.org).

As a result, an $H$. parainfluenzae (LC/1315.18) negative for $\beta$-lactamase production but resistant to penicillin, ampicillin, amoxicillin/clavulanate, cefotaxime, ceftriaxone was identified. The isolate also showed a non-susceptible profile for doxycycline and a borderline MIC for tetracycline. Fortunately, LC/1315.18 remained susceptible to carbapenems (though meropenem showed a borderline MIC of $2 \mathrm{mg} / \mathrm{L}$ ), trimethoprim/sulfamethoxazole and ciprofloxacin (Table 1). Thus, ciprofloxacin (500 mg, bid) was administered for 10 days. The new antimicrobial treatment was successful in eradicating the infection, as demonstrated by culture follow-up analysis.

Since the antibiotic resistance profile of LC/1315.18 was exceptional, WGS was conducted with both NovaSeq (Illumina Inc.) and MinION (Oxford Nanopore) platforms as previously done [5]. Assemblies of the Illumina whole-genome shotgun and Nanopore hybrid assemblies were done with SPAdes (v.3.12.0) and Canu (v1.7), respectively [6-7]. All assemblies were deposited to GenBank under BioProjects PRJNA515889 (Nanopore hybrid) and PRJNA575148 (Illumina SPAdes). AA sequences of PBP3 and PBP5 were compared to their wild type counterparts of $H$. parainfluenzae T3T1 strain (GenBank accession no. NC_015964) by implementing the protein database Protein BLAST (blastp) of NCBI. The phylogenetic tree was constructed using the tool for microbial core genome alignment and variant detection Parsnp (v1.2; http://github.com/marbl/harvest) [8]. In short, a core-genome alignment was generated with the genomes of $26 \mathrm{H}$. parainfluenzae (WGS available in the GenBank nucleotide database) and the Illumina SPAdes assembly of LC/1315.18. Finally, the FigTree software (v1.4.4; http://tree.bio.ed.ac.uk/software/figtree) was implemented to visualize the Parsnp core-genome SNV phylogenetic tree.

WGS analysis showed several point mutations in PBP3 and PBP5 sequences, thus suggesting the potential role of derived AA substitutions in determining high-level resistance to 3-GCs. Of note, PBP3 showed 98\% AA identity (Supplemental Fig. 1) with respect to the wild type $H$. 
parainfluenzae strain T3T1 (GenBank accession no. FQ312002) (i.e., Gly11Ala; Val34Met; Val356Leu; Ser385Thr; Ile442Phe; Val511Ala; Ile519Val; Asn526Lys; Asp551Leu; Thr574Ala; Phe602Leu). Instead, PBP5 displayed only 91\% AA identity (Supplemental Fig. 2) due to 37 AA substitutions scattered through the entire protein sequence. Since no $\beta$-lactamases were detected, resistance to $\beta$-lactams was reasonably attributable to the substitutions in PBP3 and/or PBP5.

Comparison with other genomes deposited in GenBank showed that LC/1315.18 had a unique profile, with no clonal relationship with other previously reported $H$. parainfluenzae strains (Supplemental Fig. 3).

We described the first 3-GC-resistant $H$. parainfluenzae strain from Italy. More importantly, the isolate showed very high MIC values for 3-GCs (the one of cefotaxime was notable: >32 mg/L) and a borderline MIC for meropenem (data not previously described in $H$. parainfluenzae). Our molecular analysis showed several AA substitutions in PBP3 and PBP5 (most of them not so far described) that are probably responsible for this phenotype. However, we could only evaluate the cumulative effect of these substitutions providing this particular resistance phenotype. As a limitation, we did not explore in vitro the effect of individual polymorphisms on the resistance to $3 \mathrm{GC}$ by cloning or site mutagenesis experiments, so not assessing which substitution could have a major role for this resistant phenotype.

The spread of resistance traits in $H$. parainfluenzae is a matter of concern. To this regard, our findings highlight that empirical prescription of antibiotics in the community setting without using microbiological data could be harmful to patients. Moreover, continuous education and awareness of general practitioners toward resistance to antibiotics, including rare phenotypes, could be of utmost importance in promoting antibiotic and diagnostic stewardship, as well in the community setting. 
101 Competing interests: None declared.

102 Funding: None.

103 Ethical approval: Not required.

104 


\section{REFERENCES}

1061 Deza G, Martin-Ezquerra G, Gómez J, Villar-García J, Supervia A, Pujol RM. Isolation of 107 Haemophilus influenzae and Haemophilus parainfluenzae in urethral exudates from men with 108 acute urethritis: a descriptive study of 52 cases. Sex Transm Infect 2016; 92: 29-31.

1092 Skaare D, Anthonisen IL, Kahlmeter G, Matuschek E, Natås OB, Steinbakk M, et al. 110 Emergence of clonally related multidrug resistant Haemophilus influenzae with penicillin111 binding protein 3-mediated resistance to extended-spectrum cephalosporins, Norway, 2006 to 112 2013. Euro Surveill 2014; 19, pii: 20986.

1133 Cerquetti M, Giufrè M, Cardines R, Mastrantonio P. First characterization of heterogeneous 114 resistance to imipenem in invasive nontypeable Haemophilus influenzae isolates. Antimicrob 115 Agents Chemother 2007; 51: 3155-61.

1164 Cherkaoui A, Diene SM, Renzoni A, Emonet S, Renzi G, François P, et al. Imipenem 117 heteroresistance in nontypeable Haemophilus influenzae is linked to a combination of altered 118 PBP3, slow drug influx and direct efflux regulation. Clin Microbiol Infect 2017; 23: 118.e9-19.

1195 Bernasconi OJ, Principe L, Viaggi V, Luzzaro F, Endimiani A. Novel vanA-carrying plasmid in 120 a clinical isolate of Enterococcus avium. Int J Antimicrob Agents 2019; 53: 876-7.

1216 Bankevich A, Nurk S, Antipov D, Gurevich AA, Dvorkin M, Kulikov AS, et al. SPAdes: a new 122 genome assembly algorithm and its applications to single-cell sequencing. J Comput Biol 2012; 19: $455-77$.

7 Koren S, Walenz BP, Berlin K, Miller JR, Bergman NH, Phillippy AM. Canu: scalable and accurate long-read assembly via adaptive $k$-mer weighting and repeat separation. Genome Res 2017; $27: 722-36$.

8 Treangen TJ, Ondov BD, Koren S, Phillippy AM. The Harvest suite for rapid core-genome 


\section{LEGEND TO THE FIGURES}

132

133 Supplemental Figure 1. NCBI protein BLAST results of PBP3 (i.e., query H. parainfluenzae 134 LC/1315.18, subject H. parainfluenzae T3T1).

135

136 Supplemental Figure 2. NCBI protein BLAST results of PBP5 (i.e., query H. parainfluenzae 137 LC/1315.18, subject $H$. parainfluenzae T3T1).

138

139 Supplemental Figure 3. Phylogenetic tree based on single nucleotide variants (SNV) of $26 H$. 140 parainfluenzae WGS available in the NCBI database and LC/1315.18 strain.

141 Tree construction was performed using Parsnp (http://github.com/marbl/harvest) with HiSeq 142 paired-end reads assembled with the genome assembly algorithm SPAdes. The parameter $-c$ was 143 used for the curated genome directory, and $-C$ for the maximal cluster D value (reference coverage) 144 was set to 5000. Remaining options were let as default. Final graphical visualization of the 145 phylogenetic relationships was created with FigTree software 146 (http://tree.bio.ed.ac.uk/software/figtree). Tip labels correspond to H. parainfluenzae strains. NA, 147 not available 